\title{
A new route to control texture of materials: Nanostructured ZnFe2O4 photoelectrodes
}

Asif Ali Tahir, Henry A. Burch, K.G. Upul Wijayantha and Bruno G. Pollet

\begin{abstract}
Studies were conducted to investigate the influence of deposition solution composition (methanol $\leq$ the deposition solvent $\leq$ ethanol) on their physical and chemical properties that matters in the aerosol formation and subsequent decomposition during the aerosol assisted chemical vapour deposition (AACVD) of $\mathrm{ZnFe}_{2} \mathrm{O}_{4}$ electrodes. The FEGSEM studies found that the change of composition of deposition solution produced a dramatic change in the $\mathrm{ZnFe}_{2} \mathrm{O}_{4}$ electrode texture. The $\mathrm{ZnFe}_{2} \mathrm{O}_{4}$ electrodes deposited from methanol as well as predominately methanolic solvents had a relatively compact morphology. In contrast, the electrodes deposited from ethanol as well as predominately ethanolic solvents showed highly textured rod-like structure at nanoscale. The change in electrode texture is explained in terms of changes occurred in precursor decomposition pathways from heterogeneous and homogeneous when the composition of deposition solution is systematically varied. The photoelectrochemical (PEC) properties of all $\mathrm{ZnFe}_{2} \mathrm{O}_{4}$ electrodes were studied by recording $J \mathrm{e} V$ characteristics under AM1.5 illumination and the photocurrent spectra. The textured electrodes exhibited a significantly higher photocurrent compared to their compact counterparts. This is attributed to the improved photogenerated minority carrier collection at the $\mathrm{ZnFe}_{2} \mathrm{O}_{4}$ /electrolyte interface as the average feature size gradually decreased. The photocurrent density (at $0.25 \mathrm{~V}$ vs. $\mathrm{Ag} / \mathrm{AgCl} / 3 \mathrm{M} \mathrm{KCl}$ ) increases rapidly when the electrode is deposited from the solvent containing $60 \%$ ethanol and above, which is in close agreement with the textural changes taken place in $\mathrm{ZnFe}_{2} \mathrm{O}_{4}$ electrodes.
\end{abstract}

\section{Introduction}

The photoelectrolysis of water using semiconductors (light harvesters as well as energy converters) is the "Holy Grail" of solar energy conversion and storage in the form of hydrogen, which is widely considered to be the sustainable solution for current energy crisis [1]. Since the pioneering work of Fujishima and Honda on photoelectrolysis on $\mathrm{TiO}_{2}$ electrodes, a considerable amount of research has been conducted [2]. Particularly, a large number of semiconductors have been studied as photoanodic materials for water splitting [3,4]. A desirable photoanodic material should have a band gap of $1.8 \mathrm{e} 2.0 \mathrm{eV}$ (in order to 
harvest sufficient amount of sunlight), be environmental-friendly, resistive to dissolution and photocorrosion, stable for prolonged exposure to sunlight, economical for mass production, and available at a low cost $[1,5,6]$. Among other key requirements, the ability to have efficient interfacial charge transfer and low recombination (at semiconductor/electrolyte interface) are key to the success of water splitting [7]. Unfortunately, many known semi-conductor photoanodes are found to suffer from poor photogenerated minority carrier collection and sluggish interfacial charge transfer reactions at the semiconductor/electrolyte interface. A significant amount of photogenerated charges seemed to lose via bulk and surface recombination at the interface. The hole collection at the semiconductor/electrolyte interface can be improved if the photoelectrode possesses a desired texture (i.e. morphology, individual feature size and shape and orientation) [8]. For example, the photoinduced charge collection at a- $\mathrm{Fe}_{2} \mathrm{O}_{3} /$ electrolyte has been enhanced by incorporating electrodes consisting of a 3-D meso-structure with individual features at nanoscale. This was done in order to minimize the bulk recombination which was believed to dominate in a- $\mathrm{Fe}_{2} \mathrm{O}_{3}$ as the charge mobility is considerably low [9]. When the electrode texture is altered in such a way that the individual feature size is controlled comparable to space charge width, the bulk recombination is reduced and the hole collection efficiency under illumination is increased. Under such conditions, a nanostructured electrode with the overall light absorption cross-section (the effective film thickness) equals to light penetration depth, can be utilized to harvest most of the incident light $[10,11]$.

To date a number of fabrication methodologies have been proposed to construct nanostructured photoelectrodes by texture control such as thermal oxidation [12], anodization [13], atmospheric pressure chemical vapour deposition (APCVD) [14,15], template synthesis [16], ultrasonic synthesis [17] and Aerosol-Assisted Chemical Vapor Deposition (AACVD) [5,18]. These methods can essentially be grouped into two paradigms: top-down and bottom-up. In particular, bottom-up methods based on soft chemical routes have attracted a considerable attention because of their relatively low cost and high throughput. The bottom-up approach refers to the build-up of materials from the substrate: atom-by-atom, molecule-bymolecule, or cluster-by-cluster. Growth species such as atoms, ions, and molecules, after impinging on the growth surface, assemble into crystal structures one after another [19]. Investigation of different physicochemical mechanisms affecting the deposition rate and the quality of end material is a subject of material chemists' interest. Among "bottom-up" chemical approaches, AACVD, a variant of CVD, enables the deposition of ultrafine, either single or multi-component material thin films with controlled stoichiometry and morphology (internal surface area, individual feature size and shape and the orientation) [20]. The AACVD process utilizes a vapourizable/ combustible aerosol to deliver the precursors to the reaction zone [18]. The formation of fine features at nanoscale is generally governed by two decomposition pathways. The first is the heterogeneous process, which is similar to the concept of making thin films by spray pyrolysis $[18,21]$. In a 
heterogeneous process, the aerosol cascades onto the heated substrate surface and the film is produced by gradual adsorption and decomposition of material on the substrate surface. The alternative pathway is the homogeneous process, in which the decomposition occurs in the vapour-phase. This process exploits the exothermic high-temperature oxidation of the reactive carrier aerosol to generate steep local temperature gradients for controlling the vapour-phase decomposition for particle formation and growth. Upon entering the high-temperature environment, the carrier aerosol droplets ignite and react exothermically. A series of processes then lead to vapourization and condensation of nanoscale particles within the thermal boundary region of the reacting droplets. The formation and growth of the nanoscale particles occur entirely within the thermal boundary region of individual droplets, making each carrier aerosol droplet effectively a micro-reactor. As a result, nanoscale particles from gaseous state plummet on the substrate surface to produce films [18]. Furthermore, AACVD is a relatively simple and versatile technique for nanostructure and texture controlled deposition of thin film electrodes. It offers significant advantages including; (i) low deposition temperatures, (ii) size-selective growth, (iii) morphological control, (iv) large scale production and (v) a single solution source can be used to fabricate multicomponent materials.

The formation of thin films in the AACVD process is typically the result of either homogeneous or heterogeneous decomposition reaction, and/or combination of both (homogeneous decomposition in the gas phase and heterogeneous growth on the substrates) [18]. When solvents with distinct physical and chemical properties that matters for the aerosol generation and subsequent decomposition (i.e. enthalpy of combustion, density and surface tension) are used as the deposition solvents, it is evident that abrupt changes take place in the target material texture [22]. This is due to the fact that those physical properties (of solvents) govern the decomposition pathways [18]. The result is losing the control over the texture significantly during the material formation. Hence, controlling the texture of a target material in a systematic manner is not possible when deposition solvents with distinctly different physical and chemical properties are used. This is a barrier that material chemists must overcome. In the present work, we have attempted to manipulate and vary the composition of deposition solution and control the precursor decomposition pathways. For that, two alcohols e.g. ethanol and methanol were chosen as the solvent combination by considering the precursor solubility. $\mathrm{ZnFe}_{2} \mathrm{O}_{4}$ was chosen to prove that it is possible to control the precursor decomposition pathways (hence the texture of it), if the composition of ethanol and methanol in the deposition solvent is conveniently altered. $\mathrm{ZnFe}_{2} \mathrm{O}_{4}$ was selected as (i) metal ferrites are an emerging class of photoelectrode materials and (ii) the changes of texture in $\mathrm{ZnFe}_{2} \mathrm{O}_{4}$ electrodes can be assessed by probing the photocurrent by employing them in a standard photoelectrochemical cell. $\mathrm{ZnFe}_{2} \mathrm{O}_{4}$ is a semiconductor that meets many desired properties generally expected from 
a prospective photoanodic material, and has the potential to be employed in water splitting cells [5,20]. Its optical band gap is slightly smaller than that of the widely studied photoanodic a-Fe $\mathrm{O}_{3}$ and therefore harvests more sunlight. In fact, in combination with $\mathrm{TiO}_{2}$ as a nanocomposite, $\mathrm{ZnFe}_{2} \mathrm{O}_{4}$ has been recently studied as an efficient light harvesting photocatalyst [23,24].

The present work reports a new method of systematically controlling the material texture by manipulating the composition of solvent combination in the AACVD deposition solvent. The results show that the strategy works for $\mathrm{ZnFe}_{2} \mathrm{O}_{4}$ when ethanol and methanol are selected as the solvent combination. This method can easily be adapted to control the texture of other materials by using suitable precursors in combination with appropriate solvent combinations. In narrow-terms, the present work reports a neat way of controlling the semi-conductor electrode texture to be employed in PEC cells. In broad-terms, this work opens up a new area of research that will trigger further exploitation to obtain desired nanostructures at controlled manner for target applications. Undoubtedly, nanostructures prepared by this method have the potential to be employed in a range of applications such as photovoltaics, gas sensors, thermoelectrics, fuel cells and catalysts.

\section{Experimental methods}

All chemicals were purchased from SigmaeAldrich. The electrodes were deposited onto fluorine-doped tin oxide (FTO) coated glass $\left(1 \times 2 \mathrm{~cm}^{2}\right)$ (TEC 8 Pilkington, 8 $\mathrm{U} / \mathrm{sq}$ ) using the single source precursor and AACVD process reported elsewhere. ${ }^{20}$ For the simplicity the AACVD precursor has been referred to as the zinc ferrite precursor in the rest of the paper. For each deposition, a $6 \mathrm{ml}$ of $0.12 \mathrm{mM}$ zinc ferrite precursor in methanol, ethanol or various compositions of methanol/ ethanol were used. The methanol/ethanol composition was altered to change the proportion in $10 \%$ (by volume) increments between pure ethanol and methanol (i.e. methanol $\leq$ the deposition solvent $\leq$ ethanol). The precursor flow rate was adjusted using flow meters and the aerosol flow was controlled by controlling the flow of carrier gas. The films were optimized by changing the gas flows, surface temperature of the hotplate, and deposition times. The changes were monitored using $\mathrm{Je} V$ curves and FEGSEM. The density corresponds to each deposition solvent (i.e. ethanol, methanol, different compositions of methanol/ethanol) was evaluated by conducting density flask measurements [25]. The surface tension of each deposition solution was determined by employing a de Nouy tensiometer [26]. The surface morphology of the $\mathrm{ZnFe}_{2} \mathrm{O}_{4}$ electrodes was studied using a Phillips XL30 field emission gun scanning electron microscope (FEG-SEM) at a beam potential of $15 \mathrm{kV}$.

The photoelectrochemical properties of the $\mathrm{ZnFe}_{2} \mathrm{O}_{4}$ electrodes (used as working electrodes) were measured using a standard three-electrode electrochemical cell fitted with a quartz window. A Pt gauze and a $\mathrm{Ag} / \mathrm{AgCl} / 3 \mathrm{M} \mathrm{KCl}$ electrodes were

\section{http://repository.uwc.ac.za}


employed as the counter and reference electrodes respectively. All measurements were carried out in $1 \mathrm{M} \mathrm{NaOH}$ aqueous electrolyte and at $298 \mathrm{~K}$. Potentiodynamic measurements were conducted using a micro autolab type-III potentiostat. The chopped currentevoltage measurements of all the $\mathrm{ZnFe}_{2} \mathrm{O}_{4}$ electrodes were recorded under AM 1.5 illumination (Solar Light, USA) within the potential range of -0.4 to po.7 $\mathrm{V}$ (vs. $\mathrm{Ag} / \mathrm{AgCl} 3 \mathrm{M} \mathrm{KCl}$ ) at a scan rate of $100 \mathrm{mV} \mathrm{s}^{-1}$.

\section{Results and discussion}

Initial studies were conducted to investigate the influence of deposition solution composition (methanol $\leq$ the deposition solvent $\leq$ ethanol) on their relevant physical and chemical properties (i.e. relative density of solvent, relative surface tension, and relative enthalpy of combustion). This was followed by the preparation of a family of $\mathrm{ZnFe}_{2} \mathrm{O}_{4}$ electrodes using deposition solvent at various methanol to ethanol ratios (methanol $\leq$ the deposition solvent $\leq$ ethanol). The resulting electrodes were then subjected to FEGSEM analysis in order to investigate their texture. The change of electrode texture was further confirmed by PEC characterization.

\subsection{The effect of composition of deposition solution on electrode textures}

Previous studies have shown that the texture of resulting thin film electrodes can be altered by employing different deposition solvents in AACVD. The solvent influences the deposition pathway by altering the aerosol droplet size and the thermodynamics of the processes taking place inside the deposition chamber [18]. The physical properties of solvent such as surface tension, density, viscosity and vapour pressure directly influence the aerosol droplet size and distribution at a constant frequency. As a result, the aerosol droplet size and distribution influence the particle size and shape, morphology and texture of the deposited materials (in powder or thin film form) $[27,28]$. Similarly, the enthalpy of combustion of the solvent also influences the morphology and texture of the deposited material by altering the chemical kinetics and thermodynamics of the processes occurring in the deposition chamber. Furthermore, the deposition solution plays a key role in determining the phase, crystallinity and composition of the deposited material [29]. The focus of the present work is to manipulate the precursor decomposition reaction pathways in AACVD by controlling aerosol droplet size as well as the enthalpy of combustion of solvent. Methanol, ethanol and their different ratios were selected as deposition solvents. Both methanol and ethanol have similar physical properties such as surface tension, viscosity and density but the enthalpy of combustion of ethanol $(-1277.17$ $\left.\mathrm{kJ} \mathrm{mol}^{-1}\right)$ is approximately twice as much as that of the methanol $(-676.15 \mathrm{~kJ}$ $\mathrm{mol}^{-1}$ ) [30].

The composition of the deposition solution was varied from methanol to ethanol by gradually adding latter to former, in $10 \%$ (by volume). The changes made in the deposition solution composition and its influence on the decomposition pathways

\section{http://repository.uwc.ac.za}


can be evaluated by understanding the effect on certain fundamental properties. Two key parameters that influenced by this compositional changes are the aerosol droplet size and the enthalpy of the combustion. Jokanovic et al. have shown that the aerosol droplets size depends upon the density and surface tension of the deposition solution at a constant frequency. In their investigations, they estimated the aerosol droplet size using Equation (1) [31].

$$
d=\left(\frac{\pi \sigma}{\rho f^{2}}\right)^{1 / 3}
$$

where, $d$ is the aerosol droplet diameter, $\mathrm{s}$ is the surface tension of the solvent, $\mathrm{r}$ is the solvent density, and $f$ is frequency of the ultrasonic oscillator. The frequency of the ultrasonic oscillator was kept constant at $40 \mathrm{kHz}$. The details of density and surface tension measurements of deposition solution with different compositions of methanol and ethanol solvent are given in supporting information (Fig. S1).

Using Equation (1) it was found that the aerosol droplet size decreased with an increase in ethanol content in the deposition solution. As shown in Fig. 1, the decrease of estimated aerosol droplet size is very small. However, combined with the enthalpy of combustion it has a pronounced effect on the texture and morphology of the resulting $\mathrm{ZnFe}_{2} \mathrm{O}_{4}$ electrodes. The enthalpy of combustion of the solvent $(\mathrm{DH})$ is a key parameter in determining the decomposition pathway in AACVD. The enthalpy of combustion of ethanol is approximately twice as much as that of the methanol. The additional energy provided by combustion of highly exothermic solvent component (in this case ethanol) can shift the decomposition pathway from a heterogeneous to a homogeneous process by aiding in the precursor decomposition [5]. The relative enthalpy of combustion vs. vol.\% ethanol in the deposition solution is given in Fig. S2 (supporting information).

The equipment for preparation of $\mathrm{ZnFe}_{2} \mathrm{O}_{4}$ electrodes consists of an ultrasonic atomizer, a transport nozzle, deposition chamber/reactor and an exhaust system [32]. In principle, two types of reactor geometry are possible: a parallel and perpendicular set-up, depending on whether the aerosol flow arrives in deposition chamber parallel or perpendicular to the substrate surface. These set ups can alter the precursor decomposition pathways as the temperature gradient in the deposition chamber is different in each case. In the present case, a perpendicular set-up was employed (Fig. 2) as the parallel set-up often results in a thickness gradient of the deposited thin film material in the flow direction [33]. In the perpendicular arrangement there is a temperature gradient from the substrate surface to the gas phase, but the aerosol travels in the opposite direction to this temperature gradient. Hence, the aerosol droplets face an increased temperature as they travel from the delivery tube end to substrate surface. The recorded 
temperature at the end of delivery tube was $65^{\circ} \mathrm{C}$ whereas it was $45^{\circ}{ }^{\circ} \mathrm{C}$ at the substrate surface. In our set-up, the distance from the end of the delivery tube to the substrate surface was maintained at $10 \mathrm{~cm}$. Typically, the aerosol droplets within this $10 \mathrm{~cm}$ distance undergo successive processes of evaporation, drying, solute precipitation, thermolysis and decomposition. Hence, the aerosol droplet size and enthalpy of combustion is capable of influencing the deposition (i.e. decomposition pathways) by controlling the:

- type of precursor species transported to the substrate surface

- reaction of precursor species on the substrate surface

- the rate of precursor species transport

- the substrate surface temperature

The schematic diagram shown in Fig. 2 describes the main processes occurring in $\mathrm{ZnFe}_{2} \mathrm{O}_{4}$ electrode preparation in the present work. As described earlier the deposition processes depend upon the aerosol droplet size and enthalpy of combustion. In low enthalpy pathway, when the aerosol droplets enter into the heated zone the solvent evaporation and vaporization of precursor starts. The vaporized precursor molecules adsorb onto the substrate surface and undergo subsequent decomposition producing a compact structure via heterogeneous process [18]. When methanol is used as the deposition solvent, due to the relatively large aerosol droplet size and low enthalpy of combustion of it, we speculate that the heterogeneous process is mainly dominant. The analysis of electrode textures by high resolution SEM shows a compact type and provides evidence for our interpretation (Fig. 4a). In contrast, when ethanol is used as the deposition solvents the relatively small droplet size and high enthalpy of combustion caused the aerosol droplets to undergo evaporation, vaporization, precipitation and thermolysis in a single step as shown in Fig. 2. This could be due to the mechanisms of heat and mass transfer inside the droplets and the surrounding gas phase leading to the formation of electrodes governed by the homogeneous deposition process.

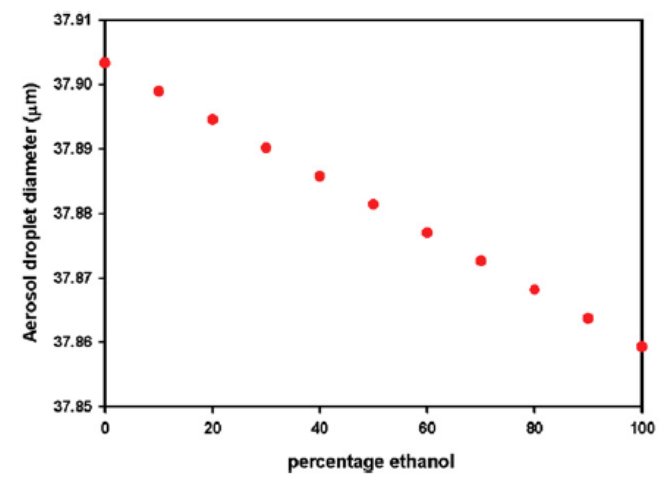

Fig. 1 - The aerosol droplet size vs. \% of ethanol in the deposition solution. 


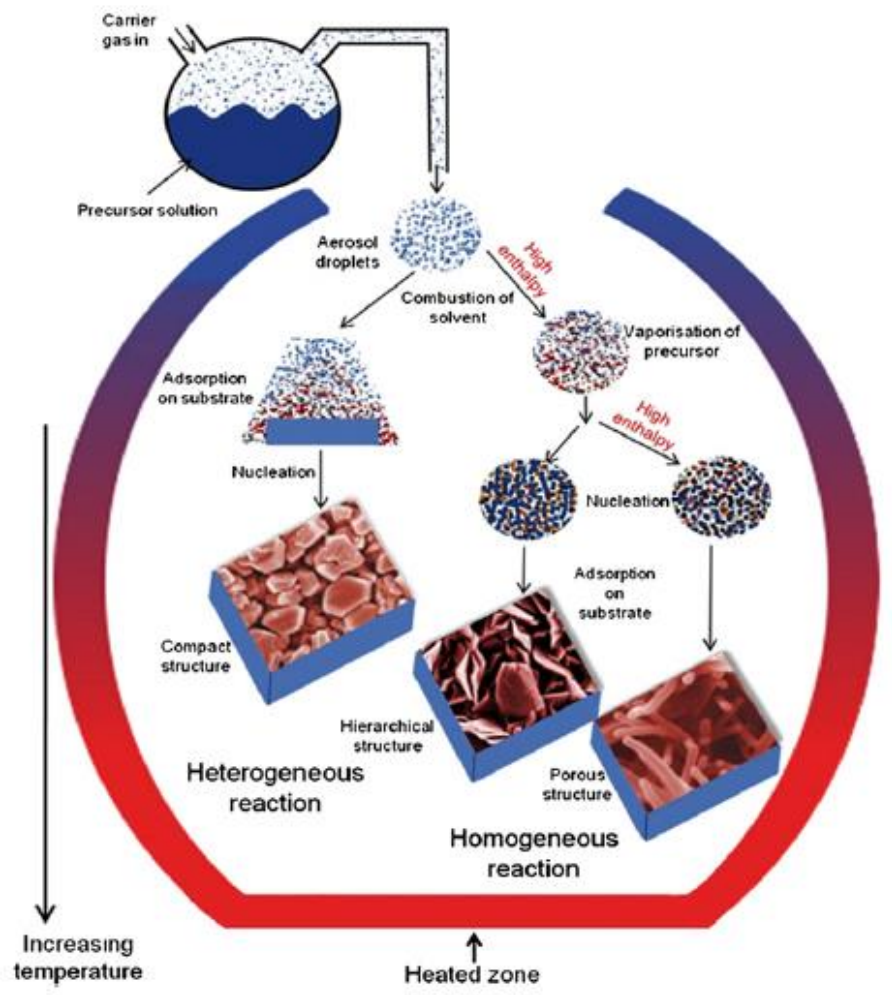

Fig. 2 - A schematic representation of homogeneous and heterogeneous reaction pathways in $\mathrm{ZnFe}_{2} \mathrm{O}_{4}$ electrode preparation.

This mechanism enables segregation suppression to the droplet scale. As a result of combustion and the decomposition that occur at the droplet level, and so-called "primary particles" arise through the thermally induced processes of nuclei formation, collision and coalescence. These particles in the gas phase undergo growth and aggregation to form the so-called "secondary particles". The secondary particle size and size distribution are mainly influenced by the properties of aerosol droplets. These primary particles and secondary particles represent either crystallites or block-mosaic assembles, which adsorbed onto the substrate surface producing highly textured nano-structured electrodes with high internal surface area [34]. The formation of $\mathrm{ZnFe}_{2} \mathrm{O}_{4}$ nanorods when ethanol was used as the deposition solvent provides ample evidence for the homogeneous deposition process (Fig. 4k). As it is evident from Fig. 4bej in the SEM topography, the deposition process gradually shifts from heterogeneous to homogenous as the vol.\% of ethanol is systematically increased in the deposition solution.

Similarly, Fig. 3 shows the effect of aerosol droplet size and enthalpy of combustion on the feature size of resulting $\mathrm{ZnFe}_{2} \mathrm{O}_{4}$ electrodes. Here, average feature size is referred to as averaged radius (in the case of non-spherical particles containing electrodes) and radius of the cross-section of nanorods (in the case of nanorod electrodes). The average $\mathrm{ZnFe}_{2} \mathrm{O}_{4}$ feature size decreases with the increase of aerosol droplet size as well as the enthalpy of combustion. The change in feature size from 
methanol to 50:50 methanol and ethanol mixture is diminutive, while there is a sharp decrease in the feature size when the vol.\% of ethanol increases beyond $50 \%$. The change is also associated with the formation of blade-like sharp edged features (Fig. 4f). Interestingly, Fig. 3 indicates that the average feature size is determined by both aerosol droplet size as well as the relative enthalpy of combustion. Further analysis of the average feature size in electrodes shows that the heterogeneous deposition process was dominant when ethanol was added up to $50 \%$ (by volume). It also shows that the reaction pathway shifted from a heterogeneous dominant to a homogeneous dominant decomposition process when further ethanol was added to the deposition solution (beyond 50\%). The sharp decrease in the $\mathrm{ZnFe}_{2} \mathrm{O}_{4}$ feature size could be possibly due to the complete decomposition of aerosol droplet in the gaseous phase and the resulting nanoparticles then adsorbed directly onto the substrate surface and act as nuclei from where the highly textured electrode grows. We believe that in case of use of ethanol as the deposition solvent the aerosol droplets decomposed close to the end of the delivery tube due to relatively small aerosol droplet size along with high heat of combustion, resulting in very fine nanoscale particles. They subsequently transfer onto the substrate surface (by carrier gas) and nucleate to from the nanorod like morphology [35].

Fig. 4 shows that the electrode morphology is systematically changed from a relatively compact (hexagonal-like) $\mathrm{ZnFe}_{2} \mathrm{O}_{4}$ particle structure to a relatively textured structure when the vol.\% of ethanol is gradually increased in the deposition solution. The relatively compact nature of the electrodes suggests that the heterogeneous deposition is the dominant reaction pathway. The change in the electrode texture and morphology is more pronounced as the vol.\% of ethanol is increased beyond $50 \%$ through a systematic change from a blade-like structure at $60 \%$ ethanol in the deposition solution. The electrode morphology shows the early signs of rod-like features when vol.\% methanol is at $80 \%$. Vertically oriented rodlike structures can be identified when $90 \%$ ethanol in the deposition solution. The electrodes deposited using $100 \%$ ethanol as the deposition solvent show further growth of nanorods. The nanorods are over $1 \mathrm{~mm}$ in length and have a random orientation on the FTO substrate surface. Based on our analysis of physical and chemical properties of deposition solution compositions, this systematic change in the electrode texture and morphology can be attributed to the change in the deposition pathway from heterogeneous to homogeneous which was influenced by both the relatively small aerosol droplet size and high heat of combustion of ethanol. The formation of nanorods from ethanol as a deposition solvent is endorsed to a complete shift of the deposition pathway to homogeneous in which completely decomposed fine $\mathrm{ZnFe}_{2} \mathrm{O}_{4}$ particles reached from gas phase to the FTO substrate surface, nucleate and then rearrange to produce nanorods. The feature size analysis (Fig. 3) and SEM topographic studies (Fig. 4) independently suggest that the deposition pathway gradually shifts from a heterogeneously 
predominant to a homogeneous predominant process with the increase of vol.\% of ethanol in the deposition solution. This is due to the considerably more exothermic enthalpy of combustion of ethanol $\left(-1277.17 \mathrm{~kJ} \mathrm{~mol}^{-1}\right)$ relative to that of methanol $\left(-676.15 \mathrm{~kJ} \mathrm{~mol}{ }^{-1}\right)$. During the deposition process the solvent combusts on approaching the heated zone (within the $10 \mathrm{~cm}$ distance between substrate surface and the end of the delivery tube). A solvent possessing a highly exothermic enthalpy of combustion provides more thermal energy for precursor decomposition and therefore aids the precursor fully decomposition in the gaseous phase itself. It is reported that a precursor that undergoes homogeneous decomposition fully decomposes before encountering the substrate and is able to form highly textured electrodes whereas a precursor that decompose through heterogeneous route produces relatively compact electrodes $[18,36]$.

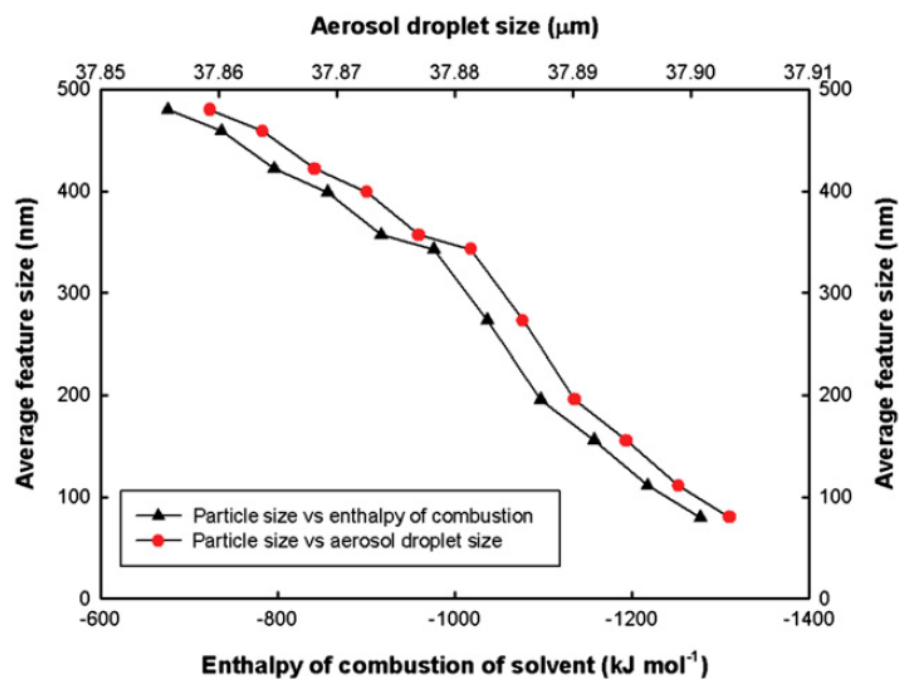

Fig. 3 - The average feature size in the electrode vs. the aerosol droplet size (red dots), and average feature size us. the relative enthalpy of combustion of the deposition solution (black triangles). (For interpretation of the references to colour in this figure legend, the reader is referred to the web version of this article.) 

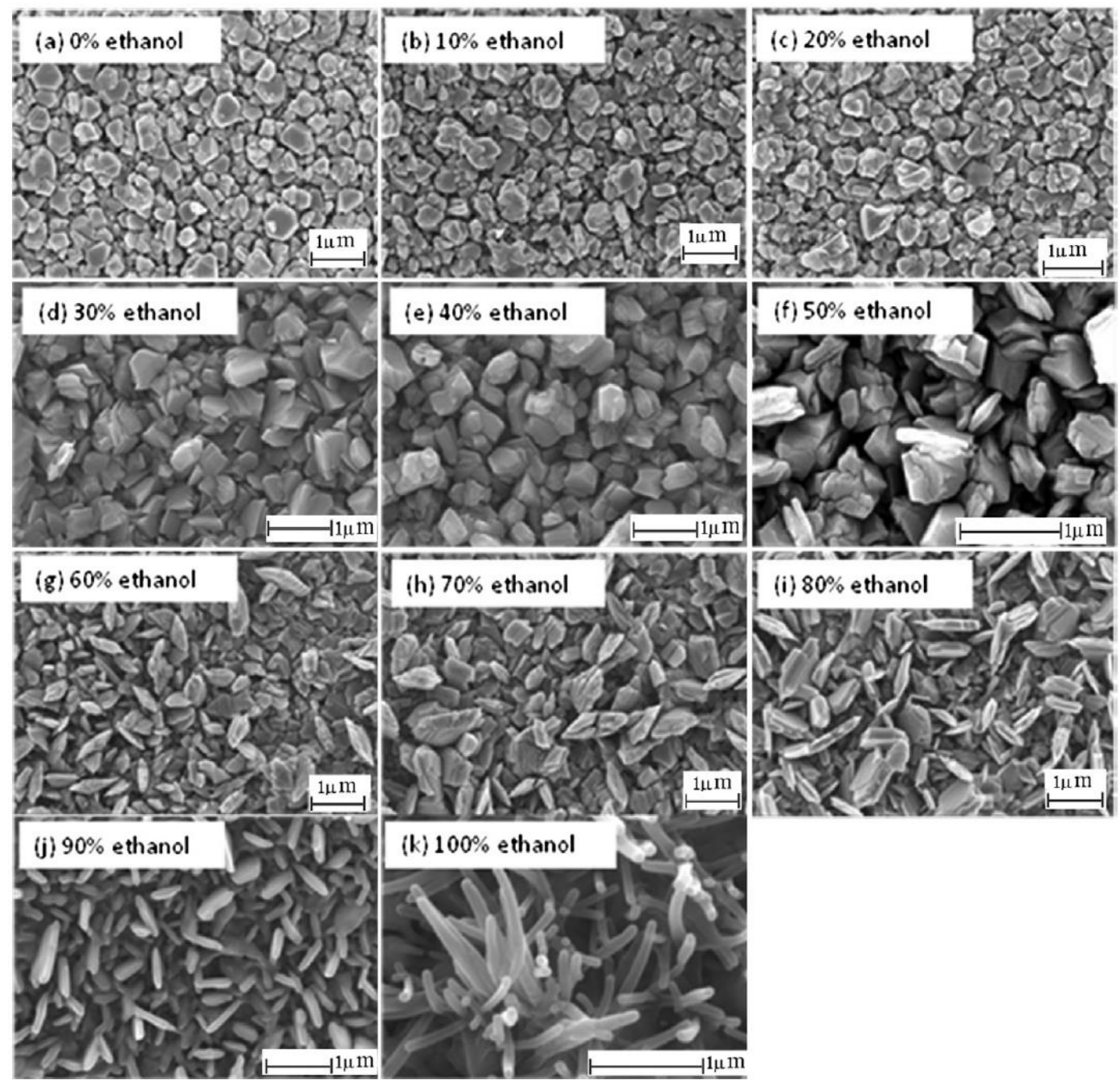

(k) $100 \%$ ethanol

Fig. 4 - The SEM topography showing changing the morphology of $\mathrm{ZnFe}_{2} \mathrm{O}_{4}$ electrodes as the vol.\% of ethanol in the deposition solution is systematically increased. (a) Shows an electrode deposited using methanol as the deposition solvent and $(\mathrm{k})$ shows an electrode using methanol as the deposition solvent. Remainder $(4 \mathrm{~b}-\mathrm{j})$ corresponds to the change in vol.\% ethanol in $10 \%$ increments.

\subsection{Photoelectrochemical properties of electrodes}

It is well-known that photoelectrochemical performance of many semiconductors suffers from poor minority carrier collection and sluggish interfacial charge transfer reactions under illumination (often referred to as 'the kinetic bottle-neck'). The hole collection can generally be improved if the photoelectrode contains a fine texture in which the feature size is comparable to hole diffusion length [8]. For example, the photoelectrochemical properties of a- $\mathrm{Fe}_{2} \mathrm{O}_{3}$ have been enhanced by incorporating materials consisting of $3-\mathrm{D}$ meso-structures with individual features at nanoscale level. This was carried out in order to minimize the bulk recombination which is believed to be dominant in materials with relatively low charge mobility [9]. When the electrode texture is modified in such a way that the individual feature size is controlled comparable to the space charge width, the bulk recombination is reduced and the hole collection efficiency increases. As we were successful in systematically controlling the texture of $\mathrm{ZnFe}_{2} \mathrm{O}_{4}$ electrodes from compact layer structure to highly textured nanostructure, it enabled us to probe the photoelectrochemical properties of all electrodes to test this hypothesis.

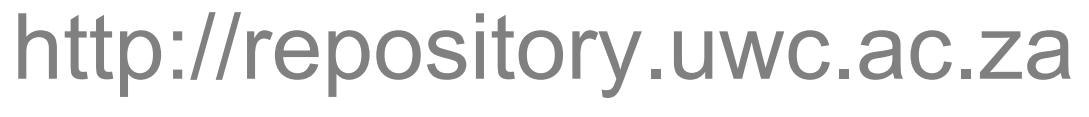


It can be anticipated based on the high resolution SEM analysis that $\mathrm{ZnFe}_{2} \mathrm{O}_{4}$ electrodes with relatively fine texture (i.e. electrodes containing nanorods) should produce superior photocurrent density compared to that of their relatively low textured counterparts (i.e. compact electrodes). Fig. 5a shows the chopped photocurrent densityevoltage plots for lowest and highest textured $\mathrm{ZnFe}_{2} \mathrm{O}_{4}$ electrodes corresponding to methanol and ethanol as deposition solvents, respectively. The textured electrodes clearly show a significantly high photocurrent density (more than doubled in the onset region). The characteristic recombination features (sharp rise and decay followed by the steady-state component in transients) in photocurrent onset region provides strong evidences for recombination occurring in that region (inset of Fig. 5a). Here, the increase of photocurrent is attributed to the decrease of individual feature size for the ethanol deposition solvent based electrode. Fig. 5b shows the photocurrent density recorded for all $\mathrm{ZnFe}_{2} \mathrm{O}_{4}$ electrodes at $0.25 \mathrm{~V}$ (vs. $\mathrm{Ag} / \mathrm{AgCl}$ reference) in $1 \mathrm{M} \mathrm{NaOH}$. The photocurrent density follows the expected trend, increasing with the decrease of average feature size of the electrode (i.e. an increase of vol.\% of ethanol in the deposition solution). It was also observed that the IPCE spectra exhibited a similar trend (data not shown here). This observation is in very good agreement with the electrode structural changes seen in SEM analyses (Fig. 4). The textured electrodes with relatively small features size seem to increase the hole collection efficiency, due to the shorter charge transfer path, and this is reflected in the photocurrent density $[10,11]$. The photocurrent rises steadily until 60 vol.\% of ethanol in the deposition solution. It increases steeply as the ethanol composition increases beyond $70 \%$, probably reflecting the fine textural changes occur beyond that solvent composition. This indirectly suggests that the hole mobility in $\mathrm{ZnFe}_{2} \mathrm{O}_{4}$ is low as in the case of a-Fe2 $\mathrm{O}_{3}$. Despite our extensive search we were unable to find the hole mobility values of $\mathrm{ZnFe}_{2} \mathrm{O}_{4}$. 

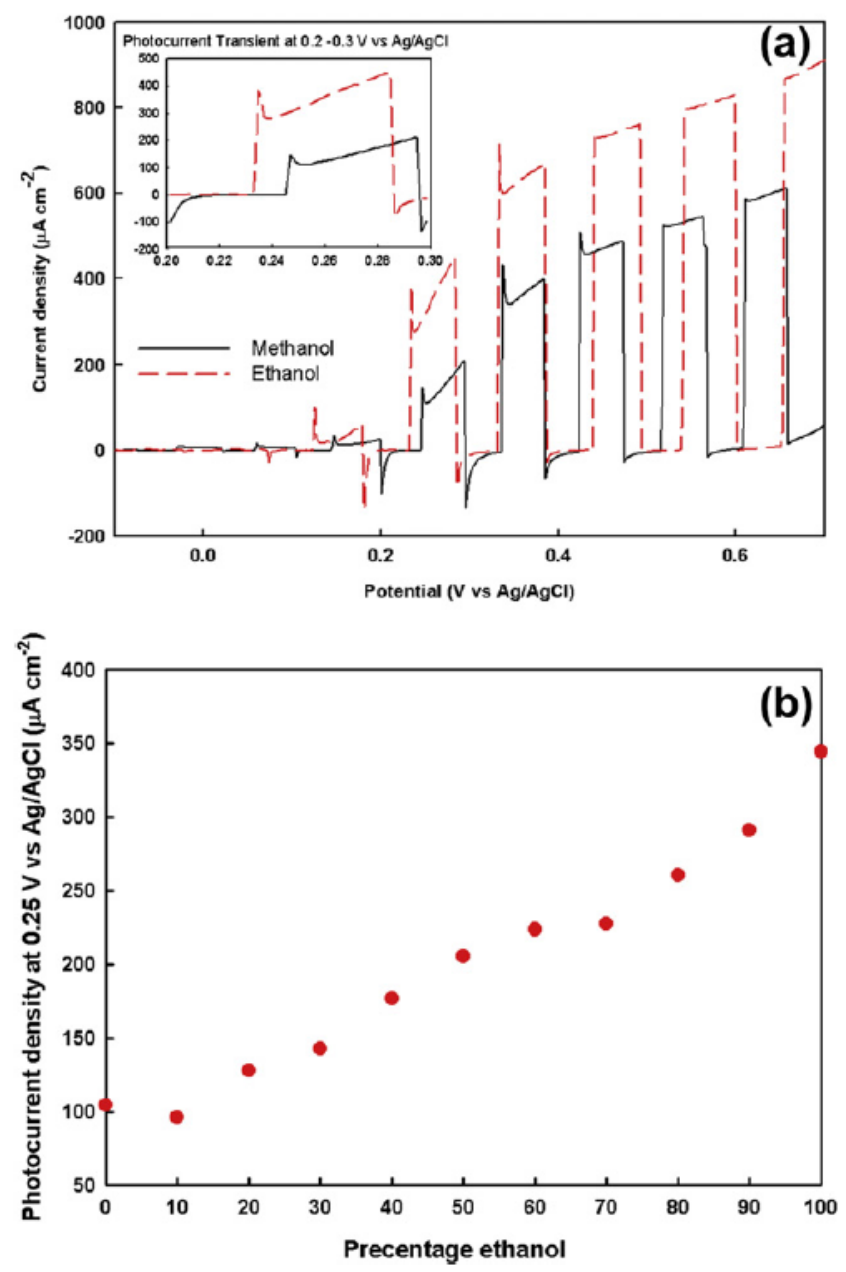

Fig. 5 - (a) The chopped J-V curves for methanol (black) and ethanol (red) deposition solvent based $\mathrm{ZnFe}_{2} \mathrm{O}_{4}$ electrodes. (b) The photocurrent density of all $\mathrm{ZnFe}_{2} \mathrm{O}_{4}$ electrodes recorded at $0.25 \mathrm{~V}$ vs. $\mathrm{Ag} / \mathrm{AgCl}$ reference in $1 \mathrm{M}$ $\mathrm{NaOH}$ us. vol.\% of ethanol in the deposition solvent. (For interpretation of the references to colour in this figure legend, the reader is referred to the web version of this article.)

\section{Conclusions}

The focus of the present work is to demonstrate a possible method for the texture, morphology, and nanostructured controlled growth of thin film electrodes to enhance the photoelectrochemical performance of $\mathrm{ZnFe}_{2} \mathrm{O}_{4}$ electrodes. The texture and morphology of the deposited $\mathrm{ZnFe}_{2} \mathrm{O}_{4}$ electrodes are controlled by systematically shifting the decomposition pathway for heterogeneous to homogeneous decomposition reactions in AACVD. The deposition pathway was tuned by manipulating the physical and chemical properties (i.e. relative density of solvent, relative surface tension, and relative enthalpy of combustion) that matters in the aerosol formation and subsequent decomposition during the AACVD of $\mathrm{ZnFe}_{2} \mathrm{O}_{4}$ electrodes. It was found from the FEGSEM studies that the changes in composition of deposition solution for $100 \%$ methanolic solution to $100 \%$ ethanolic 
solution at regular frequency (10\% by volume) produced a dramatic change in the $\mathrm{ZnFe}_{2} \mathrm{O}_{4}$ electrode textures. The $\mathrm{ZnFe}_{2} \mathrm{O}_{4}$ electrodes deposited from methanol as well as predominately methanolic solution (as the deposition solvent) had a relatively compact morphology. In contrast, the electrodes deposited from ethanol as well as predominately ethanolic solvent exhibited highly textured rod-like structure at nanoscale.

The textural changes taken place in electrodes are explained in terms of changes of precursor decomposition pathways from heterogeneous and homogeneous when the composition of deposition solution is systematically varied. The PEC properties of all $\mathrm{ZnFe}_{2} \mathrm{O}_{4}$ electrodes were studied by recording $\mathrm{JeV}$ characteristics (under AM1.5 illumination) and photocurrent spectra. As expected, the textured electrodes (deposited by employing ethanol and predominately ethanolic solvent, in the deposition solution) have shown a significantly higher photocurrent compared to their compact counterparts (deposited by employing methanol and predominately methanolic solvent, in the deposition solution). This is attributed to the improved photogenerated minority carrier collection at the $\mathrm{ZnFe}_{2} \mathrm{O}_{4}$ /electrolyte interface. Analysis of $J \mathrm{e} V$ characteristics also shows that the photocurrent density (at $0.25 \mathrm{~V}$ vs. $\mathrm{Ag} / \mathrm{AgCl} / 3 \mathrm{M} \mathrm{KCl}$ ) increases rapidly when the electrode is deposited from $60 \%$ ethanol and above (by volume) as the deposition solvent, which is in good agreement with the textural changes taken place in $\mathrm{ZnFe}_{2} \mathrm{O}_{4}$.

\section{Acknowledgements}

This work was supported by UKRC. The authors would like to thank all the members of the Renewable Energy Group of the Department of Chemistry at Loughborough University for their assistance. HAB wishes to acknowledge his studentship received through the UKRC Doctoral Training Centre in Hydrogen, Fuel Cells and their Applications. The support received from the School of Chemical Engineering at the University of Birmingham to conduct the FEGSEM studies is also acknowledged.

\section{Appendix A. Supplementary data}

Supplementary data related to this article can be found at http://dx.doi.org/10.1016/j.ijhydene.2013.01.130. 


\section{References}

[1] Van de Krol R, Liang Y, Schoonman J. Solar hydrogen production with nanostructured metal oxides. J Mater Chem 2008;18:2311e20.

[2] Fujishima A, Honda K. Electrochemical photolysis of water at a semiconductor electrode. Nature 1972;238:37e8.

[3] Batzill M. Fundamental aspects of surface engineering of transition metal oxide photocatalysts. Energy Environ Sci 2011;4:3275e86.

[4] Tahir AA, Mazhar M, Hamid M, Wijayantha KGU, Molloy KC. Photooxidation of water by $\mathrm{NiTiO}_{3}$ deposited from single source precursor $\left[\mathrm{Ni}_{2} \mathrm{Ti}_{2}(\mathrm{OEt})_{2}(\mathrm{~m}-\right.$ OEt)6(acac) 4 ] by AACVD. Dalton Trans 2009:3674e80.

[5] Tahir AA, Wijayantha KGU. Photoelectrochemical water splitting at nanostructured $\mathrm{ZnFe}_{2} \mathrm{O}_{4}$ electrodes. $\mathrm{J}$ Photochem Photobiol A Chem 2010;216:119e25.

[6] Licht S. Thermochemical solar hydrogen generation. Chem Commun 2005:4635e46.

[7] Wang H, Turner JA. Characterization of hematite thin films for photoelectrochemical water splitting in a dual photoelectrode device. J Electrochem Soc 2010;157:F173e8.

[8] Zhang Q, Cao G. Nanostructured photoelectrodes for dye-sensitized solar cells. Nano Today 2011;6:91e109.

[9] Sivula K, Formal LF, Gratzel M. Solar water splitting: progress using hematite (a$\mathrm{Fe}_{2} \mathrm{O}_{3}$ ) photoelectrodes. ChemSusChem 2011;4:432e49.

[10] Wijayantha KGU, Saremi-Yarahmadi S, Peter LM. Kinetics of oxygen evolution at a- $\mathrm{Fe}_{2} \mathrm{O}_{3}$ photoanodes: a study by photoelectrochemical impedance spectroscopy. Phys Chem Chem Phys 2011;13:5264e70.

[11] Peter LM, Wijayantha KGU, Tahir AA. Kinetics of light-driven oxygen evolution at a- $\mathrm{Fe}_{2} \mathrm{O}_{3}$ electrodes. Faraday Discuss 2012;155:309e22.

[12] Fan ZY, Wen XG, Yang SH, Lu JG. Controlled p- and n-type doping of $\mathrm{Fe}_{2} \mathrm{O}_{3}$ nanobelt field effect transistors. Appl Phys Lett 2005;87:013e113.

[13] Mohapatra SK, John SE, Banerjee S, Misra M. Water photooxidation by smooth and ultrathin a- $\mathrm{Fe}_{2} \mathrm{O}_{3}$ nanotube arrays. Chem Mater 2009;21:3048e55.

[14] Saremi-Yarahmadi S, Wijayantha KGU, Tahir AA, Vaidhyanathan B. Nanostructured a-Fe ${ }_{2} \mathrm{O}_{3}$ electrodes for solar driven water splitting: effect of doping agents on preparation and performance. J Phys Chem C 2009;113: 4768e78.

[15] Saremi-Yarahmadi S, Tahir AA, Vaidhyanathan B, Wijayantha KGU. Fabrication of nanostructured $\mathrm{a}^{-\mathrm{Fe}_{2} \mathrm{O}_{3}}$ electrodes using ferrocene for solar hydrogen generation. Mater Lett 2009;63:523e6.

[16] Liu FF, Li XY, Zhao QD, Hou Y, Quan X, Chen GH. Structural and photovoltaic properties of highly ordered $\mathrm{ZnFe}_{2} \mathrm{O}_{4}$ nanotube arrays fabricated by a facile solegel template method. Acta Mater 2009;57:2684e90.

[17] Pollet BG. The use of ultrasound for the fabrication of fuel cell materials. Int $\mathbf{J}$ Hydrogen Energy 2010;35:11986e2004. 
[18] Tahir AA, Ehsan MA, Mazhar M, Wijayantha KGU, Zeller M, Hunter AD. Photoelectrochemical and photoresponsive properties of $\mathrm{Bi}_{2} \mathrm{~S}_{3}$ nanotube and nanoparticle thin films. Chem Mater 2010;22:5084e92.

[19] Yang P, Yan R, Fardy M. Semiconductor nanowire: what's next? Nano Lett 2010;10:1529e36.

[20] Tahir AA, Wijayantha KGU, Mazhar M, McKee V. $\mathrm{ZnFe}_{2} \mathrm{O}_{4}$ thin films from a single source precursor. Thin Solid Films 2010;518:3664e8.

[21] Helble JJ. Combustion aerosol synthesis of nanoscale ceramic powders. J Aerosol Sci 1998;29:721e36.

[22] Ehsan MA, Ming HN, Misran M, Arifin Z, Tiekink ERT, Safwan AP, et al. Effect of AACVD processing parameters on the growth of greenockite (CdS) thin films using a single-source cadmium precursor. Chem Vap Deposition 2012;18: 191e200.

[23] Yuan ZH, Zhang LD. Synthesis, characterization and photocatalytic activity of $\mathrm{ZnFe}_{2} \mathrm{O}_{4} / \mathrm{TiO}_{2}$ nanocomposite. J Mater Chem 2001;11:1265e8.

[24] Yin J, Bie L-J, Yuan Z-H. Photoelectrochemical property of ZnFe2O4/TiO2 double-layered films. Mater Res Bull 2007;42: $1402 \mathrm{e} 6$.

[25] Rees JS, Jacobsen PH. Visco-elastic parameters of dental restorative materials during setting. J Dent Mater 1989;5: 41e4.

[26] Tahir AA, Wijayantha KGU, Saremi-Yarahmadi S, Mazhar M, McKee V. Nanostructured a- $\mathrm{Fe}_{2} \mathrm{O}_{3}$ thin films for photoelectrochemical hydrogen generation. Chem Mater 2009;21:3763e72.

[27] Messing GL, Zhang S-C, Jayanthi GV. Ceramic powder synthesis by spray pyrolysis. J Am Ceram Soc 1993;76: 2707e805.

[28] Okuyama K, Lenggoro IW. Preparation of nanoparticles via spray route. Chem Eng Sci 2003;58:537e47.

[29] Desai JD, Pathan HM, Min S-K, Jung K-D, Joo O-S. Preparation and characterization of iron oxide thin films by spray pyrolysis using methanolic and ethanolic solutions. Appl Surf Sci 2006;252:2251e8.

[30] CRC handbook of chemistry and physics. 44th ed. Cleveland, OH: CRC Press; 1963. p. 2582.

[31] Jokanovic V, Janackovic DJ, Uskokovic D. Influence of aerosol formation mechanism by an ultrasonic field on particle size distribution of ceramic powders. Ultrason Sonochem 1999;6: 157e69.

[32] Palgrave RG, Parkin IP. Aerosol assisted chemical vapor deposition using nanoparticle precursors: a route to nanocomposite thin films. J Am Chem Soc 2006;128:1587e97.

[33] Shaw G, Parkin IP, Pratt K, Williams DE. Control of semiconducting oxide gassensor microstructure by application of an electric field during aerosol-assisted chemical vapour deposition. J Mater Chem 2005;15:149e54.

[34] Milosevic M, Mancic L, Rabanal ME, Gomez LS, Marinkovic K. Aerosol route in processing of nanostructured functional materials. Kona-powder and particle. Powder J 2009;27:84e103. 
[35] Hsieh Y-T, Chang L-W, Chang C-C, Shih HC. Synthesis of $\mathrm{WO}_{3}$ nanorods by thermal CVD at various gas flow rates and substrate temperatures. Electrochem Solid-State Lett 2011; 14:K40e2.

[36] Hou X, Choy K-L. Processing and applications of aerosol-assisted chemical vapor deposition. Chem Vap Deposition 2006;12:583eg6. 